\title{
Wave instabilities in an anisotropic magnetized space plasma (Research Note)
}

\author{
N. S. Dzhalilov ${ }^{1,2,3}$, V. D. Kuznetsov ${ }^{2}$, and J. Staude ${ }^{1}$ \\ 1 Astrophysikalisches Institut Potsdam (AIP), An der Sternwarte 16, 14482 Potsdam, Germany \\ e-mail: JStaude@aip.de \\ ${ }^{2}$ Institute of Terrestrial Magnetism, Ionosphere and Radio Wave Propagation of the Russian Academy of Sciences (IZMIRAN), \\ Troitsk City, Moscow Region 142190, Russia \\ e-mail: [Namig;KVD]@izmiran.ru \\ 3 Shamakhy Astrophysical Observatory of the Azerbaijan Academy of Sciences (ShAO), Baku 1000, Azerbaijan
}

Received 1 April 2008 / Accepted 1 July 2008

\section{ABSTRACT}

\begin{abstract}
Aims. We study wave instability in an collisionless, rarefied hot plasma (e.g. solar wind or corona). We consider the anisotropy produced by the magnetic field, when the thermal gas pressures across and along the field become unequal.

Methods. We apply the 16-moment transport equations (obtained from the Boltzmann-Vlasov kinetic equation) including the anisotropic thermal fluxes. The general dispersion relation for the incompressible wave modes is derived.

Results. It is shown that a new, more complex wave spectrum with stable and unstable behavior is possible, in contrast to the classic fire-hose modes obtained in terms of the 13-moment integrated equations.
\end{abstract}

Key words. magnetohydrodynamics (MHD) - instabilities - plasmas - waves - turbulence - Sun: corona

\section{Introduction}

An almost collisionless, rarefied, hot, magnetized space plasma such as that of the solar corona is anisotropic and inhomogeneous, in particular in the cross-field direction (see Aschwanden 2005). There have been observations of a thermal anisotropy of $T_{\perp} / T_{\|} \sim 2-3$ in the solar wind by both Feldman et al. (1974) and Marsch et al. (1982); of large heavyion thermal anisotropies $\left(T_{\perp} / T_{\|}>100\right)$ by Kohl et al. (1998) and Cranmer et al. (1999); of protons by Cranmer et al. (1999); and of the coronal hole temperature by Dodero et al. (1998) and Antonucci et al. (2000). An opposite ion temperature relation $T_{\|}>T_{\perp}$ is also found in solar wind observations (see Marsch 2006). Due to the anisotropy in the kinetic temperatures of protons and heavy ions, the corresponding partial pressures become anisotropic, in addition to the total thermal pressure such that $p_{\perp} \neq p_{\|}$. It is now generally accepted that the observed large ion temperature anisotropies are related to the physical mechanism by which the solar corona and solar wind are heated (see Hollweg \& Isenberg 2002; Marsch 2006).

In these circumstances, it is difficult to develop a traditional hydrodynamical description of the plasma. We therefore attempt to extend the MHD approximation by considering the anisotropy of the magnetized plasma. We consider the largescale wave peculiarities that can appear in a collisionless plasma. The large-scale plasma motions are usually described by a fluid approximation, and the integrated moment equations derived from the Boltzmann-Vlasov kinetic equation are used. If collisions between particles are rare and a strong magnetic field approximation is valid, the usual MHD equations have to be replaced by other equations for which the fluid approximation is valid too. It was shown, e.g. by Grad (1949), Chew et al. (1956), and Rudakov \& Sagdeev (1958), that for a collisionless plasma
- mainly across a magnetic field - the fluid approach can be used. However, these so-called 13-moment equations cannot be used to describe a plasma of arbitrary anisotropic pressure. We therefore use the 16-moment equations.

\section{Basic equations and wave equations}

The 16-moment set of equations was used by many authors in different theoretical approaches, especially for modeling the solar wind (see Demars \& Schunk 1979; Olsen \& Leer 1999; Li 1999; Lie-Svendsen et al. 2001). The 16-moment set of transport equations for the collisionless plasma in the presence of gravity $g$ but without magnetic diffusivity is given as follows (see e.g. Oraevskii et al. 1985):

$\frac{\mathrm{d} \rho}{\mathrm{d} t}+\rho \operatorname{div} \boldsymbol{v}=0$

$\rho \frac{\mathrm{d} \boldsymbol{v}}{\mathrm{d} t}+\nabla\left(p_{\perp}+\frac{B^{2}}{8 \pi}\right)-\frac{1}{4 \pi}(\boldsymbol{B} \cdot \nabla) \boldsymbol{B}=\rho \boldsymbol{g}$

$$
+\left(p_{\perp}-p_{\|}\right)[\boldsymbol{h} \operatorname{div} \boldsymbol{h}+(\boldsymbol{h} \cdot \nabla) \boldsymbol{h}]+\boldsymbol{h}(\boldsymbol{h} \cdot \nabla)\left(p_{\perp}-p_{\|}\right),
$$

$\frac{\mathrm{d}}{\mathrm{d} t} \frac{p_{\|} B^{2}}{\rho^{3}}=-\frac{B^{2}}{\rho^{3}}\left[B(\boldsymbol{h} \cdot \nabla)\left(\frac{S_{\|}}{B}\right)+\frac{2 S_{\perp}}{B}(\boldsymbol{h} \cdot \nabla) B\right]$,

$\frac{\mathrm{d}}{\mathrm{d} t} \frac{p_{\perp}}{\rho B}=-\frac{B}{\rho}(\boldsymbol{h} \cdot \nabla)\left(\frac{S_{\perp}}{B^{2}}\right)$,

$\frac{\mathrm{d}}{\mathrm{d} t} \frac{S_{\|} B^{3}}{\rho^{4}}=-\frac{3 p_{\|} B^{3}}{\rho^{4}}(\boldsymbol{h} \cdot \nabla)\left(\frac{p_{\|}}{\rho}\right)$,

$\frac{\mathrm{d}}{\mathrm{d} t} \frac{S_{\perp}}{\rho^{2}}=-\frac{p_{\|}}{\rho^{2}}\left[(\boldsymbol{h} \cdot \nabla)\left(\frac{p_{\perp}}{\rho}\right)+\frac{p_{\perp}}{\rho} \frac{p_{\perp}-p_{\|}}{p_{\|} B}(\boldsymbol{h} \cdot \nabla) B\right]$,

$\frac{\mathrm{d} \boldsymbol{B}}{\mathrm{d} t}+\boldsymbol{B} \operatorname{div} \boldsymbol{v}-(\boldsymbol{B} \cdot \nabla) \boldsymbol{v}=0, \operatorname{div} \boldsymbol{B}=0$, 
where $\nabla=\nabla_{\|}+\nabla_{\perp}, \nabla_{\|}=\boldsymbol{h}(\boldsymbol{h} \cdot \nabla)$,

$\frac{\mathrm{d}}{\mathrm{d} t}=\frac{\partial}{\partial t}+(\boldsymbol{v} \cdot \nabla), \boldsymbol{v}=\boldsymbol{v}_{\|}+\boldsymbol{v}_{\perp}, \boldsymbol{h}=\frac{\boldsymbol{B}}{B}$,

and $S_{\|}$and $S_{\perp}$ are the heat fluxes along the magnetic field of parallel and perpendicular thermal motions. If the thermal fluxes are neglected, $S_{\perp}=0$ and $S_{\|}=0$, we obtain the equations describing the laws of the change in longitudinal and transverse thermal energy along the trajectories of the plasma (the left-hand parts of Eqs. (3) and (4)). These so-called "double-adiabatic" invariants and Eqs. (1), (2), and (7) also form a closed system of equations, the CGL (Chew-Goldberger-Low) equations (see Chew et al. 1956). By using the CGL-equations, we would however obtain incomplete equations instead of Eqs. (5), (6). This is because, by deriving the CGL equations, authors so far omitted without proof the third moments of the distribution function and therefore the thermal fluxes (see Chew et al. 1956; and Baranov \& Krasnobayev 1977). The equations derived for the 16-moment set, in our case Eqs. (1)-(7), include the thermal fluxes; they are more complete, and the CGL equations cannot be derived from these equations as a special case. For simplicity, we assume that the basic initial equilibrium state of the plasma is homogeneous ( $g=0$, and the quantities $v_{0}, \rho_{0}, p_{\perp 0}, p_{\| 0}, B_{0}, S_{\perp 0}$, and $S_{\| 0}$ are constant). Equations (1) to (7) automatically satisfy this equilibrium state with nonzero thermal fluxes. We consider small linear perturbations of all physical variables, for example pressure in the form $p=$ $p_{0}+p^{\prime}(r, t)$, where $p^{\prime}(r, t) \sim \operatorname{expi}(\boldsymbol{k} \cdot \boldsymbol{r}-\omega t), \omega$ is the wave frequency, and $k$ is the wave number. For the perturbations, we obtain linear wave equations. Even if we insert zero initial heat fluxes $S_{\| 0}=S_{\perp 0}=0$, the perturbations of these functions will never become zero: $S_{\|}^{\prime} \neq 0, S_{\perp}^{\prime} \neq 0$. Using the 16-moment equations, we should derive more reliable results about the wave properties in an anisotropic plasma than with the CGL equations based on the 13-moment equations.

In the presence of an external magnetic field, the initial collisionless heat fluxes should be defined by solutions of the kinetic equations. We should, however, use some appropriate estimate as a parameter. The heat flux functions should be estimated by taking the thermal energy density of the electrons multiplied by the particle stream speed along the magnetic field $u_{0}$ : $S_{\| 0} \approx \frac{3}{2} n_{\mathrm{e}} k_{\mathrm{B}} T_{\|} u_{0} \delta=\frac{3}{4} \delta u_{0} p_{\|}$. Hollweg $(1974,1976)$ provided some estimates of the correction parameter $\delta$ ( $\alpha$ in these papers) by assuming realistic shapes of electron distribution functions and comparing the results with space observations. We note that $\delta$ depends on the magnetic field. In the range of $B=0.1-100 \mathrm{G}$, the estimates give $\delta \approx 4-0.1$. In the same way, $S_{\perp 0} \approx \frac{3}{4} \delta u_{0} p_{\perp}$, and we define the parameter $\gamma=(3 / 4) \delta u_{0} / c_{\|}$. We note that Marsch \& Richter (1987) quoted values of $\gamma$ measured in the solar wind.

We introduce dimensionless parameters and note that the indices " 0 " of physical parameters are omitted for simplicity:

$\alpha=\frac{p_{\perp}}{p_{\|}}, c_{\|}^{2}=\frac{p_{\|}}{\rho}, \beta=\frac{B^{2}}{4 \pi p_{\|}}=\frac{v_{\mathrm{A}}^{2}}{c_{\|}^{2}}$,

$\bar{S}_{\|}=\frac{S_{\|}}{p_{\|} c_{\|}}, \overline{S_{\perp}}=\frac{S_{\perp}}{p_{\perp} c_{\|}}, l=\cos ^{2} \phi$,

where $\phi$ is the angle between wave vector and magnetic field, and the indices $\|$ and $\perp$ correspond to the values of the parameters along and across the magnetic field, respectively. We note that our $\beta$ is defined to be inversely proportional to the more commonly used plasma beta, $\beta=2 / \beta_{\text {plasma }}$. With the parameter $\gamma$ defined above, we have $\overline{S_{\|}}=\overline{S_{\perp}}=\gamma$.

In analogy with the usual MHD equations used e.g. by Somov et al. (2007), there are two independent wave branches in the plasma: waves that do not compress the plasma $(\operatorname{div} \boldsymbol{v}=0)$ and waves that compress the plasma (div $v \neq 0$ ). We restrict ourselves to the incompressible wave modes.

After inserting into the wave equations the condition of incompressibility $(\boldsymbol{k} \cdot \boldsymbol{v})=0$ and $\rho^{\prime}=0$, we obtain as usual the parametric dispersion equation. This is a polynomial equation of 6 th order in the frequency of oscillations. For the parameter $Z=\omega /\left(c_{\|} k_{\|}\right)$, this equation can be written in the form

$c_{6} Z^{6}+c_{5} Z^{5}+c_{4} Z^{4}+c_{3} Z^{3}+c_{2} Z^{2}+c_{1} Z+c_{0}=0$

$c_{0}=3(1-\alpha)\left[\alpha^{2}(l-1)+\alpha(1+l)+2(\beta-l)\right]$,

$c_{1}=2 \gamma\left[\beta(\alpha-2)+3 \alpha(1-2 l)+\alpha^{2}(4 l-3)+2 l\right]$,

$c_{2}=4 l+2 \beta(4 \alpha-5)+2 \alpha^{2}(1+3 l)+\alpha^{3}(l-1)$ $-\alpha(1+11 l), c_{3}=-2 \gamma\left[\beta(\alpha-2)+\alpha^{2}(2 l-1)-2 \alpha\right]$,

$c_{4}=6 l+\alpha(l-3)-2 l \alpha^{2}+2 \beta(2-\alpha)$,

$c_{5}=2 \gamma(2 l \alpha-2 l-\alpha), c_{6}=\alpha+l \alpha-4 l$.

All coefficients are real and, consequently, all solutions are real or conjugate complex. In the usual isotropic MHD case, only Alfvén waves with $\omega^{2}=k_{\|}^{2} v_{\mathrm{A}}^{2}$ are present, the phase velocities of which are equal to each other in both directions with respect to the magnetic field. Instead of assuming that $Z^{2}=\beta$ in the isotropic MHD, we determined now the 6th order Eq. (11) in the anisotropic case. With the heat fluxes for which $\gamma \neq 0$, odd nonzero coefficients $c_{1}, c_{3}$, and $c_{5}$ generate wave propagation velocities that depend on the direction of the magnetic field.

\section{Limiting and special cases}

In this section we investigate the most important cases of Eq. (11) which can be solved analytically.

\subsection{Parallel propagation}

In the case $l=1$ or $k=k_{\|}$, the six roots of Eq. (11) are simple. The first pair of roots corresponds to a pair of stable modes

$\omega= \pm k_{\|} c_{\|}$

The properties of these modes do not depend on the magnetic field. They are isotropic in terms of the direction of magnetic field. This implies that stable waves propagate along and against the magnetic field with the same phase velocity. In these modes, $\rho^{\prime}=0, v^{\prime}=0, B^{\prime}=0, p_{\|}^{\prime}=0$, and $S_{\|}^{\prime}=0$. However, $p_{\perp}^{\prime} \sim S_{\perp}^{\prime} \neq 0$. The restoring force for these modes is therefore $\nabla p_{\perp}$. Motions of particles across the magnetic field cause a perturbation of the plasma pressure $p_{\perp}$, but this is compensated by the generation of a heat flux. At the same time no hydrodynamic motion is produced by these modes, $(v=0)$ as $p_{\|}^{\prime}=0$. We therefore have unusual thermal waves: they are stable waves $(\operatorname{Im}(\omega)=0)$ that propagate $(\operatorname{Re}(\omega) \neq 0)$ with the parallel sound speed $c_{\|}$. Analogous to sound waves, they are not dispersive modes. The modes of this branch are named "isotropic thermal" waves. 

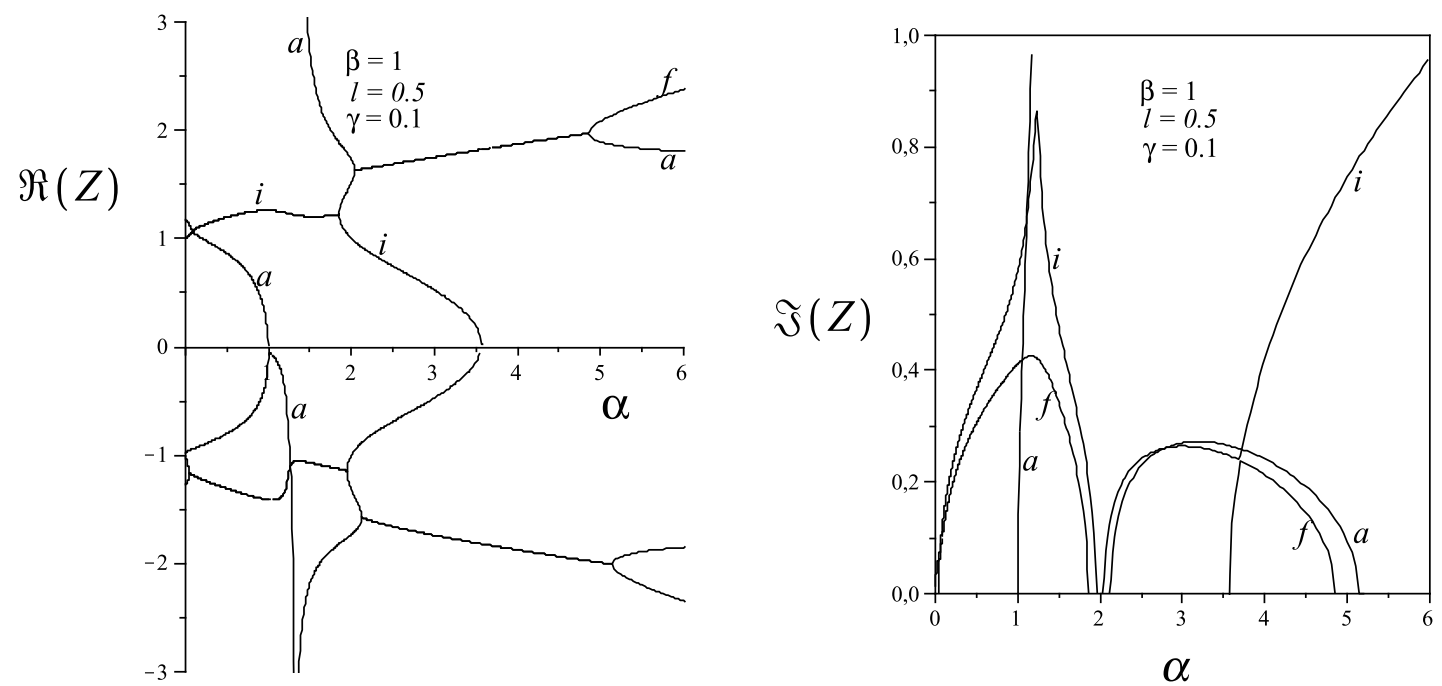

Fig. 1. Phase velocities $V_{\mathrm{ph}}=\operatorname{Re}(Z)$ of the three modes as a function of $\alpha$ for fixed parameters $\beta=1, \phi=\pi / 4$, and $\gamma=0.1$ (left picture); instability rates $\operatorname{Im}(Z)$ of the three modes (right picture). The labels at the curves correspond to modified fire-hose waves $(f)$, to isotropic thermal waves $(i)$, and to thermally anisotropic waves $(a)$.

The second pair of roots corresponds to

$\omega / k_{\|} c_{\|}= \pm \sqrt{\alpha+\beta-1}$

which are conventional isotropic fire-hose waves. The waves become unstable if $\alpha+\beta<1$ or $p_{\|}>p_{\perp}+2 p_{\text {mag }}$. The fire-hose instability therefore disappears for $\beta \geq 1$ or $\alpha \geq 1$. The dispersion relation (13) passes into that for the usual isotropic Alfvén waves $\omega^{2}=k_{\|}^{2} v_{\mathrm{A}}^{2}$ if $\alpha=1$.

The third pair of solutions corresponds to

$2 \frac{\omega}{k_{\|} \mathcal{C}_{\|}}=-\gamma \pm \sqrt{\gamma^{2}+12 \frac{1-\alpha}{2-\alpha}}$.

The instability condition $(\alpha-1) /(2-\alpha)>\gamma^{2} / 12$ is obeyed if $1<\alpha<2$. For $\gamma \neq 0$, the unstable modes begin to propagate, $\operatorname{Re}(\omega) \neq 0$. For $\gamma=0$, stable waves outside the region $1<\alpha<2$ travel along and opposite to the direction of the magnetic field with the same phase velocity. With $\gamma \neq 0$, the stable waves for which $(\alpha-1) /(2-\alpha)<\gamma^{2} / 12$ become anisotropic. Retrograde waves travel more rapidly than prograde waves. These branches are named thermally "anisotropic" waves. With increasing $\gamma$, the prograde waves propagate more slowly, but retrograde modes travel faster. There is a crossing of these branches on the axes of $\alpha$ for $\gamma=0$ at $V_{\mathrm{ph}}=0$, and for $\gamma>0$ at $V_{\mathrm{ph}}<0$. Between the crossing points of the branches and $\alpha=2$, an instability arises. In contrast to the fire-hose and the thermal modes, these modes in the instability region are traveling, $\operatorname{Re}(\omega) \neq 0$. They do not depend on $\beta$. For these waves

$\frac{p_{\|}^{\prime}}{p_{\|}}=2(1-\alpha) \frac{B^{\prime}}{B}, \quad \frac{p_{\perp}^{\prime}}{p_{\perp}}=\frac{1-\alpha^{2}}{1-2 \alpha} \frac{B^{\prime}}{B}$,

$S_{\|}^{\prime} \sim p_{\|}^{\prime}, \quad S_{\perp}^{\prime} \sim 3 \alpha \frac{1-\alpha}{1-2 \alpha} \frac{B^{\prime}}{B}$

These modes are generated by pressure anisotropy: if $\alpha=1$, they disappear.

\subsection{Oblique propagation}

\subsubsection{Strong magnetic field}

The limit $\beta \gg 1$ produces solutions of Eq. (11) that are similar to those in the parallel propagation case. In this case, the firehose waves become stable with high phase velocities, $Z^{2} \sim O(\beta)$. Other isotropic thermal and thermally anisotropic waves remain unchanged.

\subsubsection{Weak magnetic field}

In the limit $\beta \ll 1$, the first pair of solutions of Eq. (11) simplifies to $Z^{2} \approx \alpha-1$, which is the same solution as that for the parallel fire-hose waves. The other thermal modes are described by a polynomial equation of 4 th order. However, for the limit $\gamma \rightarrow 0$, we obtain

$Z^{2} \approx\left(-b_{*} \pm \sqrt{D}\right) /\left(2 a_{*}\right), \quad D=b_{*}^{2}-4 a_{*} c_{*}$,

where $c_{*}=3(1-\alpha)[\alpha(1-l)-2 l], \quad a_{*}=\alpha+l(\alpha-4)$, and $b_{*}=\alpha^{2}(1-l)-4 \alpha(1+l)+10 l$. These solutions can easily be investigated. In the region $0<\alpha<1$ for a closed area $(\alpha, l)$, we have $D<0$ and, therefore, $\operatorname{Re}(Z) \neq 0$ and $\operatorname{Im}(Z) \neq 0$. This implies that both thermal waves become unstable, and $D>0$ if $\alpha \geq 1$. In this case for unstable modes, $\operatorname{Re}(Z)=0$ and instability disappears if $\alpha \geq 2$. The thermal wave branches have crossing points, where their phase velocities coincide. For example, this occurs if $l=1$ and $\alpha=0.5$.

\subsubsection{Isotropic propagation}

We consider the important special case of arbitrary $\beta$ and $\gamma=0$. Even though the absence of fluxes, $S_{\perp}=0$ and $S_{\|}=0$, is far from reality, this simplified case was investigated using the 13-moment equations (e.g. Kato et al. 1966; Baranov \& Krasnobayev 1977; and Kuznetsov \& Oraevskii 1992). Substituting $\gamma=0$ into Eq. (11), we obtain a cubic equation for $\zeta=Z^{2}$ :

$c_{6} \zeta^{3}+c_{4} \zeta^{2}+c_{2} \zeta+c_{0}=0$. 
We have symmetrically only three pairs of solutions, $Z= \pm \sqrt{\zeta}$. The analytical solutions $\zeta_{1}, \zeta_{2}$, and $\zeta_{3}$ allow us to investigate in significant detail the dependence of the solutions on the parameters $\alpha, \beta$, and $\phi$. For a small deviation of the propagation angle $(\phi \neq 0)$, the situation differs significantly from that of the parallel propagation. All three modes interact, and this interaction occurs in two ranges of $\alpha: \alpha>\alpha_{\mathrm{c}}$ and $\alpha<\alpha_{\mathrm{c}}$. The critical value of $\alpha=\alpha_{\mathrm{c}}=4 l /(1+l)$ is the singular point for $c_{6}=0$. With increasing propagation angle, the interaction domains expand. In these domains, the growing rates also become larger and we obtain a mixture of modes - a turbulent wave motion.

\subsubsection{Anisotropic propagation}

To investigate the role of the thermal parameter $\gamma$, we solved the 6th order polynomial equation, choosing more realistic values of $\gamma<1$. In Fig. 1, the phase velocities and instability rates are shown. We observe that

a) waves with positive and negative velocities are different and all three wave branches become coupled;

b) maxima of the instability rate strongly depend on $\gamma$.

For some parameters, all three modes become unstable.

\subsection{Classic fire-hose modes}

To compare our results with the classic fire-hose modes based on the CGL equations, we use the initial set of equations, omit Eqs. (5) and (6), and substitute into the others $S_{\|}=S_{\perp}=0$. These CGL equations for incompressible waves provide two pairs of solutions:

$Z= \pm \mathrm{i} \sqrt{A}, \quad A=\frac{(1-\alpha)[\alpha(1+l)-4 l]+2 \beta(2-\alpha)}{\alpha(1+l)-4 l}$.

The fire-hose instability condition is that $A>0$. In the parallel propagation case $(l=1)$, this condition is the most familiar case of $\alpha+\beta<1$. We obtain the same result for $l \neq 1$, if $\beta=0$. The instability condition is more complete for the oblique propagation case if $\beta>0$, but this strongly differs from our results based on the 16-moment equations. For the classic fire-house instability, we always have that $\operatorname{Re}(\omega)=0$. In our case, $\operatorname{Re}(\omega) \neq 0$ in most cases due to the coupling of these modes with other thermal modes. For the classic modes in the relations, for example, $p_{\|}^{\prime} / p_{\|}=n_{1} B^{\prime} / B$ and $p_{\perp}^{\prime} / p_{\perp}=n_{2} B^{\prime} / B$, the coefficients are $n_{1}=-2, n_{2}=1$. In our case, these coefficients are complete functions of all parameters. The main difference in our case compared to that for the CGL equations is the appearance of two additional thermal branches, even if $\gamma=0$.

\section{Conclusion}

To investigate the peculiarities of large-scale wave motions in a collisionless magnetized plasma, we have applied the 16-moment transport equations, derived as integrated moments of the kinetic equations. In earlier similar attempts, the 13-moment equations were used. However, these equations exclude without any reason the thermal fluxes and are therefore incomplete.

Anisotropy is the main feature of a collisionless plasma with a strong magnetic field. In the present study, the pressure anisotropy was described by the parameter $\alpha$ and the heat fluxes by $\gamma$. By assuming that $\gamma=0$ we were unable to derive the 13moment equations, or by assuming that both $\alpha=1$ and $\gamma=0$ we did not obtain the isotropic MHD case. The 16-moment equations were in principle different equations. Using these equations, we illustrated that a wide unstable and stable wave spectrum in the collisionless anisotropic plasma was possible, even in the incompressible approximation. If $\gamma \neq 0$ (heat fluxes are present), the waves propagated along and against the magnetic field at different speeds. This behavior differed from that of the usual isotropic MHD case. The coupled wave spectrum, including modified fire-hose modes, strongly depends on the magnetic field value (parameter $\beta$ ), pressure anisotropy parameter $\alpha$, heat flux parameter $\gamma$, and wave propagation angle $\phi$ with respect to the magnetic field. The deduced instability increments are rather large. We have derived the general instability condition for incompressible waves.

Acknowledgements. We are vary thankful to Bernhard Kliem, Gottfried Mann, and the referee whose critical comments helped to improve an earlier version of this paper. The present work has been supported by the German Science Foundation (DFG) under grant No. 436 RUS 113/931/0-1 (R) which is gratefully acknowledged.

\section{References}

Aschwanden, M. J. 2005, Physics of the Solar Corona. An Introduction with Problems and Solutions, 2nd edition (Springer), 892

Antonucci, E., Dodero, M. A., \& Giordano, S. 2000, Sol. Phys., 197, 115

Baranov, V. B., \& Krasnobayev, K. V. 1977, Hydrodynamics of Cosmic Plasma (Moscow: Nauka), 335

Chew, G. F., Goldberger, M. L., \& Low, F. E. 1956, Proc. Roy. Soc. London A, 236, 112

Cranmer, S. R., Kohl, J. L., Noci, G., et al. 1999, ApJ, 511, 481

Demars, H. G., \& Schunk, R. W. 1979, J. Phys. D, 12, 1051

Dodero, M. A., Antonucci, E., Giordano, S., \& Martin, R. 1998, Sol. Phys., 183, 77

Feldman, W. C., Asbridge, J. A., Bame, S. J., \& Montgomery, M. D. 1974, Rev. Geophys. Space Phys., 12, 715

Grad, H. 1949, Commun. Pure Appl. Math., 2, 331

Hollweg, J. V. 1974, J. Geophys. Res., 79, 3845

Hollweg, J. V. 1976, J. Geophys. Res., 81, 1649

Hollweg, J. V., \& Isenberg, P. A. 2002, J. Geophys. Res., 107, 1147

Kato, Y., Tajiri, M., \& Taniuti, T. 1966, J. Phys. Soc. Japan, 21, 765

Kohl, J. L., Noci, G., Antonucci, E., et al. 1998, ApJ, 501, L127

Kuznetsov, V. D., \& Oraevskii, V. N. 1992, Astron. Lett., 18, 547

Li, X. 1999, J. Geophys. Res., 104, 19773

Lie-Svendsen, O., Leer, E., \& Hasteen, V. H. 2001, J. Geophys. Res., 106, 8217

Marsch, E. 2006, Living Rev. Sol. Phys., 3, 1

Marsch, E., \& Richter, A. K. 1987, Ann. Geophys., 5 A(2), 71

Marsch, E., Mühlhäuser, K.-H., Schwenn, R., et al. 1982, J. Geophys. Res., 87, 52

Olsen, E. L., \& Leer, E. 1999, J. Geophys. Res., 104, 9963

Oraevskii, V. N., Konikov, Y. V., \& Chazanov, G. V. 1985, Transport processes in anisotropic near-Earth plasma (Moscow: Nauka)

Rudakov, L. I., \& Sagdeev, R. Z. 1958, in The plasma physics and problems of controlling nuclear reaction (Moscow) 3, 268

Somov, B. V., Dzhalilov, N. S., \& Staude, J. 2007, Astron. Lett., 33, 309 